\title{
Velocimetry, Cooling and Rotation Sensing by Cold-Atom Matterwave Interferometry
}

\author{
M. Carey, D. Elcock, J. Saywell, S. Jones, M. Belal, T Freegarde \\ School of Physics \& Astronomy, University of Southampton, Highfield, Southampton SO17 1BJ, UK \\ tim.freegarde@soton.ac.uk
}

\begin{abstract}
Interferometric measurement of an atom's velocity allows, by tailoring the impulse imparted by the matterwave-splitting laser pulses, a velocity-dependent force that cools an atomic sample. Differential measurement reveals the sample's acceleration and rotation.
\end{abstract}

OCIS codes: (020.1335) Atom optics; (020.3320) Laser cooling; (120.5790) Sagnac effect; (120.7250) Velocimetry

\section{Interferometric velocimetry}

Raman matterwave interferometry [1] commonly uses pairs of laser beams, similar in frequency but opposite in wavevector, to couple atomic states of similar electronic configuration and energy but different linear momentum. The momentum difference gives the radiative interaction a velocity-dependence, which may be overcome if the interaction is limited to pulses short enough to be broad in bandwidth, but which persists between pulses in the phase drift between the atomic superposition and the laser oscillator. The small frequency difference between the coupled states means that there is negligible spontaneous emission, and that the beam pair can be produced with phase precision by radio-frequency modulation of a single master laser. With these ingredients, atom interferometry has been shown to be a precise means of inertial measurement [2-4].

The evolution of the wavefunction of an atom, as it moves with respect to, but between the pulses of, an interacting optical field, may be viewed from two perspectives. From that of the optical apparatus, the coupled atomic states differ in kinetic energy, and the phase of their superposition therefore accrues because of the difference in classical action between their trajectories. Viewed from the inertial frame of the atom, the phase of the optical field varies as the apparatus moves and presents different points for the stationary atom to sample. The result is a relative phase $\varphi$ between the atomic superposition and the optical field which, if the field is resonant for a stationary atom, depends after a time $t$ upon the atom's velocity $\mathbf{v}$ :

$$
\varphi(\mathbf{v}, t)=\mathbf{k}_{\text {eff }} \cdot\left(\mathbf{v}+\mathbf{v}_{\mathrm{R}} / 2\right) t
$$

where $\mathbf{k}_{\text {eff }}$ is the effective wavevector of the Raman field, equal to the wavevector difference between its components, and $\mathbf{v}_{\mathrm{R}}$ is the recoil velocity $\hbar \mathbf{k}_{\mathrm{eff}} / m$ for atoms of mass $m$.

Whether regarded as interference of the de Broglie matterwave, or of the optical wave transferred via an atomic resonator, the interference fringes of a two-pulse Ramsey interferometer show a sinusoidal dependence of the transferred population upon time, with a periodicity determined by the velocity component along $\mathbf{k}_{\text {eff. }}$ Single velocity components may be found from the fringes' periodicity, and distributions from their Fourier transform.

\section{Interferometric cooling}

Interferometric measurement of the atomic velocity is accompanied by a radiative impulse, for atoms receive an impulse of $\hbar \mathbf{k}_{\text {eff }}$ when they are transferred between the two interferometer states. By tailoring the interferometer period and introducing an adjustable phase between the two interferometer pulses, it may be arranged that this impulse on average opposes the atom's velocity, and an atomic sample is thus cooled [5]. This process, which is not in principle limited to Raman transitions, shows similar cooling rates and limiting temperatures to conventional Doppler cooling, but allows adjustment of the capture range for higher temperatures without saturating the transition for lower velocities; Raman transitions and pulsed repumping allow sub-Doppler temperatures to be reached without the usual Sisyphus mechanisms. Using stimulated Raman transitions between the ground hyperfine states, we have demonstrated the $1 \mathrm{D}$ cooling of a freely moving cloud of ${ }^{85} \mathrm{Rb}$ atoms from 21 to $3 \mu \mathrm{K}$ [6].

As originally proposed [5], interferometric cooling includes a third laser pulse to invert the atomic population between the two interferometer pulses. This has two useful effects. Firstly, it eliminates the dependence of the interferometer phase upon the detuning of the Raman field from the atom's electronic transition, by arranging that both components of the interferometer superposition spend equal times in the two atomic states. Secondly, provided that its effective wavenumber is opposite to that of the interferometer pulses, the intermediate pulse increases both the sensitivity to velocity and the accompanying impulse [7], with potential enhancement of the cooling rate [8]. 


\section{Accelerometry, gravimetry and rotation sensing}

If the intermediate pulse instead has the same effective wavenumber as the interferometer pulses, its effect is to eliminate also the dependence upon a steady velocity: the intermediate pulse is equivalent to two interferometer pulses, and the three-pulse sequence hence resembles two identical Ramsey interferometers that act upon the coupled atomic states first directly, and then as if they were interchanged. The sequence thus performs a differential measurement, revealing the change in the velocity component during the sequence. This may result from a change in the magnitude of the vector velocity under linear acceleration, or a change in its direction under rotation. Whereas linear acceleration changes all vector velocities equally, rotation changes them in proportion to their magnitudes, as characterized by the Coriolis acceleration.

Atomic point-source interferometry $[9,10]$ uses time-of-flight mapping of the velocities of atoms within a small cloud onto their positions after the cloud has expanded, to produce an interferometric fringe pattern whose spacing, orientation and offset from the cloud's origin allow two rotation components and one linear acceleration component to be determined. The finite initial size of the atom cloud blurs the fringe pattern, reducing its visibility, but should not affect the deduced rotation rates. Correlations between the initial positions and velocities of atoms within the cloud, however, cause systematic distortion of the observed fringe pattern [10]. We are currently developing such an expanding-cloud interferometric rotation sensor, and investigating coherent read-out enhancement using Raman memory techniques $[11,12]$.

\section{Composite pulse techniques for fidelity enhancement}

The use of a single inversion pulse to eliminate the dependence upon the Raman detuning is a simple example of the composite pulse technique originally developed for NMR spectroscopy [13]. More complex sequences of radiative interactions, each corresponding to partial rotations about different axes through a Bloch sphere, can be designed to compensate for systematic variations in the speed and trajectory of coherent operations that occur when different molecules or atoms see different field intensities, coupling strengths or detunings. Intensity and magnetic field gradients, distributions over Zeeman sub-states and uncorrected Doppler shifts all cause such systematic perturbations in atom interferometry. Simple composite pulse methods have already been applied to atom interferometry to increase the interferometer area and sensitivity $[14,15]$.

We have explored the performance of several established NMR pulse sequences when applied to velocitysensitive Raman state manipulation in a freely expanding cold atom cloud, allowing the inversion infidelity to be halved [16]. The agreement between experimental measurements and simple predictions shows the underlying coherence of the atomic ensemble. We are now developing pulse sequences that are specifically tailored to the variations, correlations and sensitivities of atom interferometers.

\section{References}

[1] M. Kasevich, S. Chu, “Atomic interferometry using stimulated Raman transitions,” Phys. Rev. Lett. 67, 181-184 (1991).

[2] K. Bongs, R. Launay, M. A. Kasevich, "High-order inertial phase shifts for time-domain atom interferometers,” Appl. Phys. B 84, 599-602 (2006).

[3] T. L. Gustavson, P. Bouyer, M. A. Kasevich, "Precision rotation measurements with an atom interferometer gyroscope,” Phys. Rev. Lett. 78, 2046-2049 (1997).

[4] T. L. Gustavson, A. Landragin, M. A. Kasevich, "Rotation sensing with a dual atom-interferometer Sagnac gyroscope," Class. Quantum Grav. 17, 2385-2398 (2000).

[5] M. Weitz, T. W. Hänsch, “Frequency-independent laser cooling based on interferometry,” Europhys. Lett. 49, $302-308$ (2000).

[6] A. Dunning et al., "Interferometric laser cooling of atomic rubidium," Phys. Rev. Lett. 115, 073004 (2015).

[7] J. M. McGuirk, M. J. Snadden, M. A. Kasevich, “Large area light-pulse atom interferometry,” Phys. Rev. Lett. 85, 4498-4501 (2000).

[8] T. Freegarde, G. Daniell, D. Segal, “Coherent amplification in laser cooling and trapping,” Phys. Rev. A 73, 033409 (2006).

[9] S. M. Dickerson et al., "Multiaxis inertial sensing with long-time point source atom interferometry,” Phys. Rev. Lett. 111, 083001 (2013).

[10] G. W. Hoth, B. Pelle, S. Riedl, J. Kitching, E. A. Donley, "Point source atom interferometry with a cloud of finite size," Appl. Phys. Lett. 109, 0714113 (2016)

[11] K. F. Reim et al., “Towards high-speed optical quantum memories,” Nat. Photon. 4, 218-221 (2010). 
[12] S. D. Jenkins, T. Zhang, T. A. B. Kennedy, "Motional dephasing of atomic clock spin waves in an optical lattice,” J. Phys. B 45, 124005 (2012).

[13] M. H. Levitt, “Composite pulses" in eMagRes (Wiley, 2007).

[14] D. L. Butts et al., "Efficient broadband Raman pulses for large-area atom interferometry,” J. Opt. Soc. Am. B 30, $922-927$ (2013).

[15] P. Berg et al., “Composite-light-pulse technique for high-precision atom interferometry,” Phys. Rev. Lett. 114, 063002 (2015).

[16] A. Dunning et al., “Composite pulses for interferometry in a thermal cold atom cloud,” Phys. Rev. A 90, 033608 (2014). 